\title{
Predictive value of T2-weighted magnetic resonance imaging for the prognosis of patients with mass-type breast cancer with peritumoral edema
}

\author{
TING LIANG, BIN HU, HONGWEN DU and YILI ZHANG \\ Imaging Center, The First Affiliated Hospital of Xi'an Jiaotong University \\ School of Medicine, Xi'an, Shaanxi 710061, P.R. China
}

Received February 12, 2020; Accepted September 11, 2020

DOI: $10.3892 / \mathrm{ol} .2020 .12177$

\begin{abstract}
The aim of the present study was to investigate the role of edema surrounding breast cancer masses in the prognostic prediction of magnetic resonance imaging (MRI) T2-weighted fat suppression sequence. For this purpose, 80 patients with mass-type breast cancer underwent conventional plain breast MRI, dynamic contrast-enhanced (DCE)-MRI or diffusion-weighted MRI scan. The associations between edema around the mass on MRI T2 fat suppression sequence plain scan and tumor stage, pathological findings, immunohistochemical findings and axillary lymph node metastasis were analyzed. The results revealed the presence of edema around the mass on the MRI T2 fat suppression sequence plain scan in 35 patients. By contrast, there was no abnormal enhancement on the DCE-MRI, and the apparent diffusion coefficient value did not decrease in these areas. Compared with the remaining 45 patients, the 35 patients with peritumoral edema exhibited a higher tumor stage and a higher rate of axillary lymph node metastasis (all $\mathrm{P}<0.05)$. However, there was no significant difference in pathological classification or the expression of estrogen receptor, progesterone receptor and human epidermal growth factor receptor 2 (as determined by immunohistochemistry) between the two groups. In total, 12 cases of tumor shrinkage during neoadjuvant chemotherapy were accompanied by an improvement in edema. Taken together, the findings of the present study indicated that the presence of edema around the mass on the MRI T2 fat suppression sequence may predict poor prognosis in patients with mass-type breast cancer. Furthermore, the improvement of the peritumoral edema
\end{abstract}

Correspondence to: Dr Yili Zhang, Imaging Center, The First Affiliated Hospital of Xi'an Jiaotong University School of Medicine, 277 Yanta West Road, Xi'an, Shaanxi 710061, P.R. China

E-mail: yilizhang1975@hotmail.com

Key words: edema, magnetic resonance imaging, breast cancer, prognosis, predictive value post-neoadjuvant chemotherapy may also be a predictor of a more favorable prognosis.

\section{Introduction}

Breast magnetic resonance imaging (MRI) is widely used in clinical practice. The methods of MRI examination include the conventional plain scan, the dynamic contrast-enhanced (DCE) scan and the diffusion-weighted imaging (DWI) scan. DCE-MRI plays an important role in the diagnosis of breast lesions, with a reported sensitivity of $95-99 \%$ in detecting invasive cancer and $80 \%$ in detecting ductal carcinoma in situ, whereas its mean reported specificity is $80 \%$ (range, 37-97\%) (1-5). Moreover, the curve of the DCE scan may indicate the enhancement type of the lesions. DWI is a functional MRI technique that characterizes tissues by their water diffusion properties. DWI and the apparent diffusion coefficient (ADC) value have been used successfully in the differential diagnosis between malignant and benign breast diseases, and to determine tumor extension. It has been reported that the combination of DCE-MRI with DWI has a sensitivity of $95.7 \%$ and a specificity of $89.2 \%$; therefore, radiologists mainly base their diagnosis on the combination of these two techniques (6).

As regards plain scans, the signal of the lesions is relatively similar to that of normal tissue in the conventional $\mathrm{T} 1$ sequence and the T2 fat suppression sequence; therefore, some lesions may be easily overlooked without DCE-MRI as radiologists may not examine the plain scan more closely. On this note, recent studies have suggested that T2-weighted images are a valuable component of the MRI evaluation of edema $(7,8)$. It has been reported that peritumoral edema is a common imaging manifestation of advanced or inflammatory breast cancer. The cause of edema is associated with the poor drainage caused by lymphatic obstruction. For dense breasts, it is often difficult to distinguish edema from tumor lesions on mammography. However, the sequence of the plain scan and enhanced scan may help distinguish between tumors and edema.

The aim of the present study was to compare the prognostic value of the MRI T2 fat suppression sequence with that of the DCE-MRI and DWI scan in patients with mass-type 
breast cancer exhibiting peritumoral edema. The associations between the presence of edema and tumor stage, pathological classification, immunohistochemical findings and axillary lymph node metastasis were further investigated in order to determine the clinical prognostic value of the MRI T2 fat suppression sequence in breast cancer. In addition, the association between the improvement of edema and tumor shrinkage in patients who underwent neoadjuvant chemotherapy (NAC) was also investigated.

\section{Materials and methods}

Patients. A total of 80 patients (age range, 24-77 years; mean age \pm standard deviation, $45.1 \pm 11.1$ years) with histologically proven breast cancer who had undergone an MRI examination for tumor staging and detection of axillary lymph node involvement between September 2017 and December 2018 were included in the present study. The study protocol was approved by the Ethics Committee of the First Affiliated Hospital of Xi'an Jiao Tong University (no.: XJTU1AF2017LSK-72), and all patients provided written informed consent prior to their inclusion in the study. The MRI examination was performed in the second week of the menstrual cycle in premenopausal women. No breast implants were found in any of the patients. Histopathological diagnosis was performed following surgical resection in 27 cases and puncture biopsy in 53 cases.

Immunohistochemical staining. Breast tumor tissues were fixed in formalin $(10 \%, 24 \mathrm{~h}$, room temperature) embedded in paraffin and cut into $5-\mu \mathrm{m}$ sections. The sections were deparaffinized and immersed in citrate buffer $(\mathrm{pH} 6.0)$ for $5 \mathrm{~min}$ to retrieve antigen, followed by $0.3 \%$ hydrogen peroxide for $15 \mathrm{~min}$ to block endogenous peroxidase activity. The sections were then blocked for $30 \mathrm{~min}$ using $10 \%$ goat plasma (Invitrogen; Thermo Fisher Scientific, Inc.). Following incubation with primary antibodies directed against estrogen receptor (ER; ab32063; 1:1,000), progesterone receptor (PR; ab16661; 1:400) or human epidermal growth factor receptor 2 (HER2; ab214275; 1:4,000) for $1 \mathrm{~h}$ at $37^{\circ} \mathrm{C}$, the slides were incubated with an HRP-conjugated goat anti-rabbit secondary antibody (ab205718; 1:2,000) according to the manufacturer's recommendations. All primary and secondary antibodies were purchased from Abcam. Finally, the sections were visualized with diaminobenzidine and counterstained with hematoxylin for $5 \mathrm{~min}$ at room temperature, then dehydrated in alcohol and xylene and mounted onto glass slides. The numbers of ER-, PR- or HER2-positive cells were counted from at least 10 randomly selected fields of each sample (NIKON ECLIPSE T1-S; Nikon Corporation) at a magnification of $x 400$. The tissue samples were classified as positive for ER and PR when the proportion of positively stained tumor cells was $>1 \%$ (9). For HER2, the tumor was considered positive if $>10 \%$ of the tumor cells exhibited positively stained nuclei (10).

MRI protocol. MRI examinations were performed on a 3.0 T MR device (Discovery 750w, Cytiva) using a four-channel breast coil. The protocol consisted of the following: Transverse T2 FSE-IDEAL ASSET sequence [TR/TE=6020/80.7 $\mathrm{msec}$, field of view $(\mathrm{FOV})=320 \times 320 \mathrm{~mm}(\mathrm{AP} \times \mathrm{RL})$, matrix 256x256, 4-mm slice thickness and 1-mm gap]; transverse T1-weighted TSE [TR/TE=867/5.7 $\mathrm{msec}$, FOV=320x320 mm (AP x RL), matrix 256x256, 4-mm slice thickness and 1-mm gap]; 3D dynamic, CE T1-weighted vibrant sequences [TR/TE=7.6/4.3 msec, FOV=320x320 mm (AP x RL), matrix $125 \times 125,5-\mathrm{mm}$ slice thickness without gaps, 6 dynamic acquisitions, a dynamic data acquisition time of $1 \mathrm{~min}$ and $30 \mathrm{sec}$, and a total sequence duration of $9 \mathrm{~min}$ ]; gadobenate dimeglumine (MultiHance ${ }^{\circledR}$ ), which is considered as the most accurate contrast medium used in this field, was intravenously injected at a dose of $0.1 \mathrm{mmol} / \mathrm{kg}$ body weight and flow rate of $2 \mathrm{ml} / \mathrm{sec}$ followed by $10 \mathrm{ml}$ of saline solution, as previously reported (11); EPI-DWI sequences were acquired in the transversal orientation using the following parameters: TR/TE=4,000/69.6 msec, FOV=320x320 mm (AP x RL), matrix 128x140, 4-mm slice thickness with 1-mm gap.

Image analysis. All MRI data were transferred to and analyzed on a diagnostic workstation equipped with dedicated software for MRI examination (Advantage Workstation 4.6; Cytiva). Two radiologists with 5 years of experience in the field of breast MRI who were blinded to the clinical findings and pathological diagnosis reviewed all the MRI films. In addition to the size, shape, signal, enhancement curve and ADC value of the mass, the presence of edema around the tumor mass, skin thickening and increase of subcutaneous fibrous connective tissue on the MRI T2 fat suppression sequence were more closely observed. Moreover, the presence of abnormal enhancement on dynamic enhanced scanning and increased signal intensity on DWI on these areas were also determined.

Statistical analysis. The $\chi^{2}$ test or Fisher's exact test were used to analyze the association between the MRI T2 fat suppression sequence findings around the mass and tumor stage, pathological classification, luminal classification and axillary lymph node metastasis. All calculations were performed using SPSS software, version 25.0 (IBM Corp.).

\section{Results}

Imaging characteristics. In the present study, according to the Breast Imaging Reporting and Data System Magnetic Resonance Imaging (BI-RADS-MRI) by the American College of Radiology, 80 patients were classified as type a, b, c and $d(n=10, n=31, n=25$ and $n=14$, respectively) on the basis of breast composition, and as type a, b, $c$ and $d(n=22, n=34, n=16$ and $n=8$, respectively) on the basis of background parenchymal enhancement. On the DCE-MRI, 3 patients exhibited a type I curve, while 66 exhibited the type II and 11 the type III curve. The ADC value of 16 cases was $>1.2 \times 10^{-3} \mathrm{~mm}^{2} / \mathrm{sec}$ and that of 64 cases was $<1.2 \times 10^{-3} \mathrm{~mm}^{2} / \mathrm{sec}$. In total, 27 cases were diagnosed with BI-RADS V categories and 53 cases were diagnosed with BI-RADS VI categories.

Presence of edema. Of the 80 patients, 35 exhibited edema (Fig. 1) around the mass on the MRI T2 fat suppression sequence (group I) and the remaining 45 cases did not exhibit edema (group II). Furthermore, in group I, 26 cases exhibited skin thickening and increased subcutaneous fibrous connective 

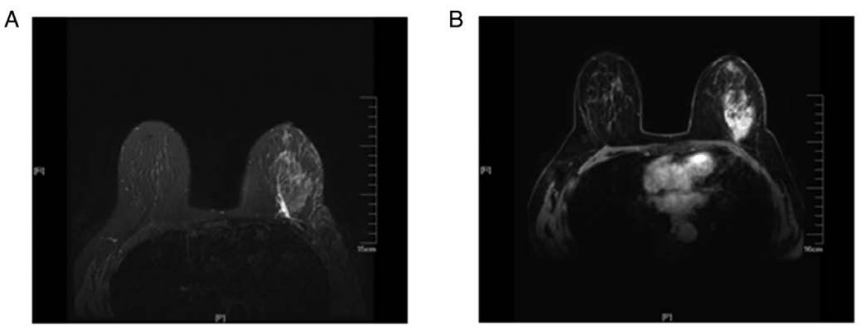

Figure 1. Magnetic resonance imaging examination of a 62 -year-old woman with invasive ductal carcinoma of the left breast with metastasis to the right axillary lymph nodes confirmed by biopsy. (A) The T2-weighted fat suppression sequence revealed the presence of edema around the tumor mass and under the skin. (B) The transverse contrast-enhanced dynamic sequence revealed that there was a markedly irregular enhancement mass in the lateral quadrant at the level of the nipple.
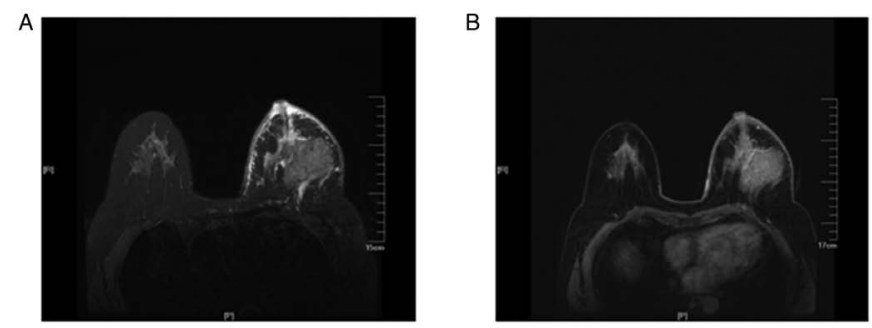

Figure 2. Magnetic resonance imaging examination of a 56-year-old woman with invasive ductal carcinoma in the left breast. (A) The T2-weighted fat suppression sequence revealed that the areola and breast skin were thickened and there was edema anterior to the pectoralis major muscle and under the skin. (B) The transverse contrast-enhanced dynamic sequence revealed that there was a mass with markedly irregular enhancement in the lateral quadrant at the level of the nipple.

tissue (Fig. 2) and 7 cases exhibited nipple retraction on the MRI T2 fat suppression sequence. Compared with the edema observed on the plain scan, there was no abnormal enhancement on the DCE and no abnormal signal on the DWI in the edema area. A total of 12 patients in group I and 3 patients in group II eventually received NAC. In the 12 cases in group 1, following chemotherapy, the size of the tumors was smaller compared with that prior to treatment, and the edema shadows around the mass, thickened skin, as well as the increased subcutaneous fibrous connective tissue on the MRI T2 fat suppression sequences also improved (Fig. 3).

Pathological classification. Pathological results were obtained by needle puncture or surgical biopsy in all 80 patients. In group I, 25 cases were non-specific invasive carcinoma and the remaining 10 cases were non-specific invasive carcinoma with high-grade ductal carcinoma. In group II, 30 cases were non-specific invasive carcinoma, 11 cases were non-specific invasive carcinoma with high-grade ductal carcinoma, 3 cases were ductal carcinoma in situ and 1 case was medullary carcinoma. There was no significant difference in pathological classification between the two groups $(\mathrm{P}>0.05)$.

Association between imaging findings and stage. According to the TNM staging criteria of breast cancer, the tumors were classified as stage I, II, III and IV. The associations between the MRI T2 fat suppression sequence imaging findings around
Table I. Correlation between the findings of magnetic resonance imaging T2 fat suppression sequence around the mass and tumor stage.

\begin{tabular}{lrrrrr}
\hline & \multicolumn{5}{c}{ Tumor stage } \\
\cline { 2 - 6 } Groups & I & II & III & IV & P-value \\
\hline I $^{\text {a }}$ & 3 & 3 & 25 & 4 & 0.000 \\
II $^{\text {b }}$ & 9 & 33 & 3 & & \\
\hline
\end{tabular}

${ }^{\mathrm{a} P r e s e n c e ~ o f ~ p e r i t u m o r a l ~ e d e m a . ~}{ }^{\mathrm{b}} \mathrm{Absence}$ of peritumoral edema.
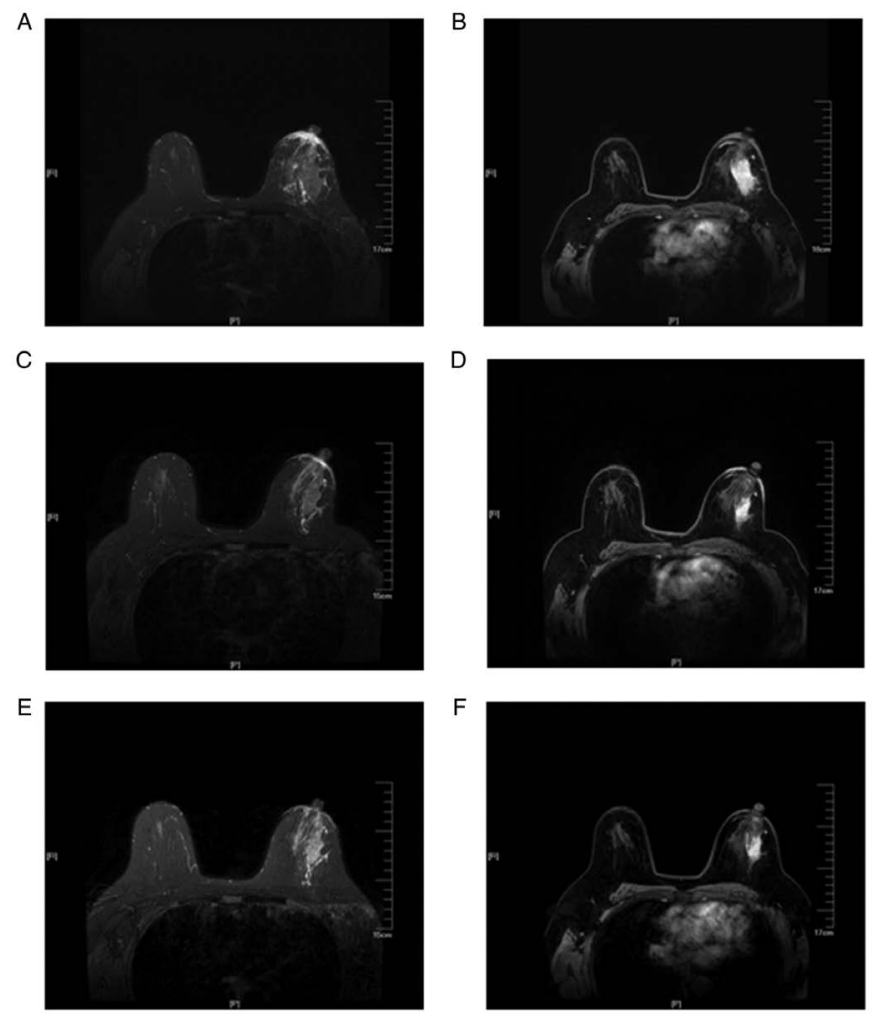

Figure 3. Magnetic resonance imaging examination of a 47-year-old woman with invasive ductal carcinoma in the left breast who received NAC. (A) The T2-weighted fat suppression sequence revealed the presence of edema around the tumor mass and areola, with breast skin thickening prior to NAC. (B) Transverse contrast-enhanced dynamic sequence revealed that there was a mass exhibiting markedly irregular enhancement in the lateral quadrant at the level of the nipple prior to NAC. (C and E) The T2-weighted with fat suppression sequence revealed that the presence of edema around the mass and areola, as well as the breast skin thickening had improved after (C) 2 and (E) 4 cycles of NAC. (D and F) Contrast-enhanced dynamic sequences revealed that the mass was significantly smaller compared with that prior to NAC after (D) 2 and (F) 4 cycles of NAC. NAC, neoadjuvant chemotherapy.

the mass and tumor stage are presented in Table I. Compared with group II, the patients in group I exhibited a significantly higher tumor stage $(\mathrm{P}<0.05)$.

Association between imaging findings and hormone receptor status. According to the expression of ER, PR and HER2 on immunohistochemistry (Fig. 4), the tumors were categorized as luminal A, luminal B, HER2 ${ }^{+}$and triple-negative. The associations between the MRI T2 fat suppression sequence imaging 
Table II. Correlation between the findings of magnetic resonance imaging T2 fat suppression sequence around the mass and luminal type.

\begin{tabular}{lcccc}
\hline & \multicolumn{3}{c}{ Luminal type } \\
\cline { 2 - 5 } Groups & Luminal A & Luminal B & HER2 ${ }^{+}$ & Triple-negative \\
\hline $\mathrm{I}^{\mathrm{a}}$ & 5 & 13 & 6 & 11 \\
$\mathrm{II}^{\mathrm{b}}$ & 5 & 27 & 6 & 7 \\
\hline
\end{tabular}

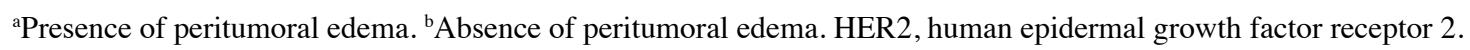
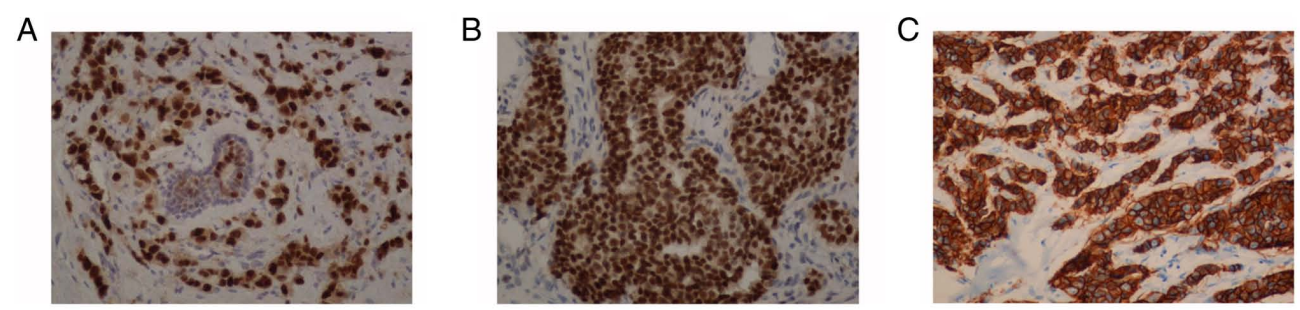

Figure 4. Immunohistochemical staining of ER, PR and HER2 in breast tumor tissues at a magnification of $x 200$. Strong immunostaining for (A) ER, (B) PR and (C) HER2 in breast tumor tissue. ER, estrogen receptor; PR, progesterone receptor; HER2, human epidermal growth factor receptor 2.

findings around the mass and luminal type are presented in Table II. There was no significant difference in the expression of ER, PR or HER2, as determined by immunohistochemistry, between the two groups $(\mathrm{P}>0.05)$.

Association between imaging findings and axillary lymph node metastasis. All 80 patients underwent axillary lymph node biopsy or surgery. The associations between the MRI T2 fat suppression sequence imaging findings around the mass and axillary lymph node metastasis are presented in Table III. Compared with group II, the patients in group I exhibited a higher rate of axillary lymph node metastasis $(\mathrm{P}<0.05)$.

\section{Discussion}

MRI is the most sensitive method for the detection of breast diseases. The DCE-MRI and DWI techniques are hotspots in current research and the main focus is the study of the tumor per se. By contrast, for regular base sequences, the research on the T1 plain scan and T2 fat suppression sequence has been less extensive. In particular, in recent years, studies have demonstrated that there is no significant difference between fast magnetic resonance scanning and conventional scanning in the diagnosis of lesions; the only exception is that the time required for the former is markedly shorter (12-14). Thus, the conventional $\mathrm{T} 2$ fat suppression sequence was deleted from the fast magnetic resonance sequence. The present study aimed to determine whether the $\mathrm{T} 2$ fat suppression sequence can provide other information that may be valuable for the diagnosis of breast cancer.

The clinical and imaging data of 80 cases of mass-type breast cancer were collected for the present study. In 35 of those cases, grid or patchy blurred high signal shadows were identified around the mass on the T2-weighted fat suppression sequence, whereas there was no grid or patchy blurred high signal shadows around the mass in the other 45 cases. However, no abnormal enhancement or diffusion was found in these areas on DCE-MRI or DWI.

A previous study indicated that the aforementioned abnormal signal on the T2-weighted with fat suppression sequence was edema (15). Mammary edema refers to the swelling caused by the accumulation of fluid in the tissues of the breast. The underlying causes are usually malignant, including advanced breast cancer, inflammatory breast cancer, lymphatic inflammatory metastasis and lymphoma. Pathologically, edema caused by malignant lesions may be observed as lymphatic obstruction and/or capillary and venule obstruction (16-18). Previous studies demonstrated that peritumoral edema is likely to occur due to tumor angiogenesis, which is one of the hallmarks of cancer, and leads to increased vascular permeability and the release of peritumoral cytokines $(19,20)$.

On imaging, edema is characterized by increased density on mammography or signal intensity on the T2-weighted with fat suppression sequence, enlargement of fibrous trabeculae, thickening of Cooper's ligament and cutaneous edema. In contrast to T1-weighted sequences, which highlight anatomic detail and are used on the enhanced scan, fluid-sensitive T2-weighted sequences depict edema, hemorrhage, mucus, or cystic fluid (8). Therefore, T2-weighted images are a valuable component of the MRI evaluation of edema $(15,21)$.

Previous studies have demonstrated that the prognosis of breast cancer is closely associated with tumor state, luminal type and axillary lymph node metastasis $(22,23)$. It was previously demonstrated that the prognosis of patients with luminal A type breast cancer was the superior to that of patients with luminal B type breast cancer, whereas patients with HER2-positive and triple-negative breast cancer had the worst prognosis (24). Further research found that patients with HER2-positive and luminal B breast cancer were more 
Table III. Correlation between the findings of magnetic resonance imaging T2 fat suppression sequence around the mass and axillary lymph node metastasis.

\begin{tabular}{lccc}
\hline & \multicolumn{2}{c}{ Axillary lymph node metastasis } & \\
\cline { 2 - 3 } Groups & Metastasis & No metastasis & P-value \\
\hline $\mathrm{I}^{\mathrm{a}}$ & 17 & 18 & 0.043 \\
$\mathrm{II}^{\mathrm{b}}$ & 12 & 33 & \\
\hline
\end{tabular}

apresence of peritumoral edema. ${ }^{\mathrm{b}}$ Absence of peritumoral edema.

prone to developing multiple lesions and axillary lymph node metastasis, while the distant metastasis rate of patients with triple-negative breast cancer was significantly higher compared with that of patients with luminal A, luminal B and HER2-positive breast cancer $(25,26)$.

In the present study, the association of peritumoral edema with clinical stage, pathological classification and axillary lymph node metastasis was analyzed. The results revealed that the 35 patients with peritumoral edema had a more advanced clinical stage and higher rate of axillary lymph node metastasis compared with the remaining 45 patients; however, there was no significant difference in pathological classification or the expression of ER, PR and HER2, as determined by immunohistochemistry, between the two groups. In addition, of the 35 cases with edema, 26 exhibited skin thickening and increase of subcutaneous fibrous connective tissue, and 7 exhibited nipple retraction.

It has been reported that the presence of peritumoral edema may be a strong prognostic indicator for lymphatic spread and the cancerous infiltration of lymph nodes $(27,28)$. Cheon et al demonstrated that the presence of peritumoral edema was associated with the characteristics of biologically aggressive tumors (29) and Uematsu et al demonstrated that peritumoral edema was associated with prognostic factors (30). The results of the present study confirmed these previous conclusions, indicating the prognostic value of peritumoral edema in breast cancer. More importantly, in the present study, the shrinkage of the tumors in patients receiving NAC was accompanied by the improvement of the edema around the mass and the skin thickening, as well as a decrease of the subcutaneous fibrous connective tissue on the MRI T2 fat suppression sequences. To the best of our knowledge, few studies to date have investigated the imaging changes associated with the presence of edema around the mass following NAC. Based on these findings, less or no edema post-NAC may be used as a predictive indicator of a better prognosis.

In conclusion, the pathological basis of edema around the breast cancer mass is the infiltration of lymphatic vessels by tumor tissue, and the results of the present study demonstrated that the MRI T2 may clearly demonstrate the signs of peritumoral edema. The results further confirmed that the occurrence of edema was closely associated with a higher tumor stage and axillary lymph node metastasis. Moreover, it was observed that, with the reduction of the tumor volume following NAC, edema also improved. Therefore, the presence of peritumoral edema in the MRI T2 may be used as a valuable imaging index for evaluating the prognosis and treatment efficacy for patients with breast cancer.

However, the present study had several limitations. First, the number of cases in the study was insufficient and, therefore, the association among the three parameters (tumor stage, luminal type and axillary lymph node metastasis) was not analyzed. Second, a proportion of the patients in the present study underwent MRI following core-needle biopsy, and the peritumoral signals may have thus been affected by the post-procedural changes. Third, this study lacked further follow-up.

In conclusion, the T2-weighted images are irreplaceable for evaluating edema, skin thickening and the increase in subcutaneous fibrous connective tissue. Peritumoral edema in the MRI T2 fat suppression sequence may predict a poor prognosis in patients with mass-type breast cancer, whereas the improvement of peritumoral edema post-NAC may predict a more favorable prognosis.

\section{Acknowledgements}

Not applicable.

\section{Funding}

The present study was supported by grants from the Shaanxi Research and Development Program (grant no. 2017SF-020) and the Institutional Foundation of the First Affiliated Hospital of Xi'an Jiaotong University (grant no. 2016MS-05).

\section{Availability of data and materials}

All data generated or analyzed during this study are included in this published article.

\section{Authors' contributions}

TL analyzed and interpreted the experimental data and contributed to the writing of the manuscript. BH performed the immunohistochemical staining of the breast tumors. HD designed the experiments and reviewed and revised the manuscript. YZ designed the experiments and is the major contributor to writing the manuscript. All the authors have read and approved the final manuscript.

\section{Ethics approval and consent to participate}

The study protocol was approved by the Ethics Committee of the First Affiliated Hospital of Xi'an Jiao Tong University (no.: XJTU1AF2017LSK-72), and all patients provided written informed consent prior to their inclusion in the study.

\section{Patient consent for publication}

Not applicable.

\section{Competing interests}

All the authors declare that they have no competing interests. 


\section{References}

1. Kuhl CK: MRI of breast tumors. Eur Radiol 10: 46-58, 2000.

2. Huang W, Fisher PR, Dulaimy K, Tudoria LA, O'Hea B and Button TM: Detection of breast malignancy: Diagnostic MR protocol for improved specificity. Radiology 232: 585-591, 2004

3. Belli P, Costantini M, Bufi E, Magistrelli A, La Torre G and Bonomo L: Diffusion-weighted imaging in breast lesion evaluation. Radiol Med 115: 51-69, 2010 (In English and Italian).

4. Peter NH, Rinkes IH, Zuithoff NP, Mali WP, Moons KG and Peeters PH: Meta-Analysis of MR imaging in the diagnosis of breast lesions. Radiology 246: 116-124, 2008.

5. Moschetta M, Telegrafo M, Rella L, Capolongo A, Ianora AA and Angelelli G: MR evaluation of breast lesions obtained by diffusion-weighted imaging with background body signal suppression(DWIBS) and correlations with histological findings. Magn Reson Imaging 32: 605-609, 2014.

6. Kul S, Cansu A, Alhan E, Dinc H, Gunes G and Reis A: Contribution of diffusion-weighted imaging to dynamic contrast-enhanced MRI in the characterization of breast tumors. AJR AM J Roentgenol 196: 210-217, 2011.

7. Guo Y, Tang WJ, Kong QC, Liang YY, Han XR, Zheng BJ, Sun L, Wei XH, Jin Z and Liu CL: Can whole-tumor apparent diffusion coefficient histogram analysis be helpful to evaluate breast phyllode tumor grades? Eur J Radiol 114: 25-31, 2019.

8. Westra C, Dialani V, Mehta TS and Eisenberg RL: Using T2-weighted sequences to more accurately characterize breast masses seen on MRI. AJR Am J Roentgenol 202: W183-W190, 2014.

9. Hammond ME, Hayes DF, Dowsett M, Allred DC, Hagerty KL, Badve S, Fitzgibbons PL, Francis G, Goldstein NS, Hayes M, et al: American society of clinical oncology/college of American pathologists guideline recommendations for immunohistochemical testing of estrogen and progesterone receptors in breast cancer. J Clin Oncol 28: 2784-2795, 2010.

10. Wolff AC, Hammond ME, Schwartz JN, Hagerty KL, Allred DC, Cote RJ, Dowsett M, Fitzgibbons PL, Hanna WM, Langer A, et al: American Society of clinical oncology/college of American pathologists guideline recommendations for human epidermal growth factor receptor 2 testing in breast cancer. Arch Pathol Lab Med 131: 18-43, 2007.

11. Martincich L, Faivre-Pierret M, Zechmann CM, Corcione S, van den Bosch HC, Peng WJ, Petrillo A, Siegmann KC, Heverhagen JT, Panizza P, et al: Multicenter, double-blind, randomized, intraindividual crossover comparison of gadobenate dimeglumine and gadopentetate dimeglumine for Breast MR imaging (DETECT Trial). Radiology 258: 396-408, 2011.

12. Jain M, Jain A, Hyzy MD and Werth G: FAST MRI breast screening revisited. J Med Imaging Radiat Oncol 61: 24-28, 2017.

13. Mango VL, Morris EA, Dershaw DD, Abramson A, Fry C, Moskowitz CS, Hughes M, Kaplan J and Jochelson MS Abbreviated protocol for breast MRI: Are multiple sequences needed for cancer detection? Eur J Radiol 84: 65-70, 2015.

14. Heacock L, Melsaether AN, Heller SL, Gao Y, Pysarenko KM, Babb JS, Kim SG and Moy L: Evaluation of a known breast cancer using an abbreviated breast MRI protocol: Correlation of imaging characteristics and pathology with lesion detection and conspicuity. Eur J Radiol 85: 815-823, 2016.

15. Uematsu T: Focal breast edema associated with malignancy on T2-weighted images of breast MRI: Peritumoral edema, prepectoral edema, and subcutaneous edema. Breast Cancer 22: 66-70, 2015.
16. Kwak JY, Kim EK, Chung SY, You JK, Oh KK, Lee YH, Kwon TH and Jung HK: Unilateral breast edema: Spectrum of etiologies and imaging appearances. Yonsei Med J 46: 1-7, 2005.

17. Tekbas G, Oguzkurt L, Gurel K, Ozkan U, Gur S and Onder H: Is unilateral breast enlargement always a sign of cancer? Hemodial Int 15: 553-538, 2011.

18. Bakırköy Tıp Dergisi: Radiologic findings of breast edema and causes of unilateral edema. Med J Bakirköy 1: 1-6, 2006

19. Baltzer PA, Yang F, Dietzel M, Herzog A, Simon A, Vag T, Gajda M, Camara O and Kaiser WA: Sensitivity and specificity of unilateral edema on T2w-TSE sequences in MR-mammography considering 974 histologically verified lesions. Breast J 16: 233-239, 2010.

20. Cheon H, Kim HJ, Kim TH, Ryeom HK, Lee J, Kim GC, Yuk JS and Kim WH: Invasive breast cancer: Prognostic value of peritumoral edema identified at preoperative MR imaging. Radiology 287: 68-75, 2018.

21. Kuhl CK, Klaschik S, Mielcarek P, Gieseke J, Wardelmann E and Schild HH: Do T2 weighted pulse sequences help with the differential diagnosis of enhancing lesions in dynamic breast MRI? J Magn Reson Imaging 9: 187-196, 1999.

22. Simon SD, Bines J, Werutsky G, Nunes JS, Pacheco FC, Segalla JG, Gomes AJ, Van Eyll MH, Gimenes DL, Crocamo S, et al: Characteristics and prognosis of stage I-III breast cancer subtypes in Brazil: The AMAZONA retrospective cohort study. Breast 44: 113-119, 2019.

23. Hashmi AA, Aijaz S, Khan SM, Mahboob R, Irfan M, Zafar NI, Nisar M, Siddiqui M, Edhi MM, Faridi N and Khan A: Prognostic parameters of luminal A and luminal B intrinsic breast cancer subtypes of Pakistani patients. World J Surg Oncol 16: 1, 2018.

24. Kast K, Link T, Friedrich K, Petzold A, Niedostatek A, Schoffer O, Werner C, Klug SJ, Werner A, Gatzweiler A, et al: Impact of breast cancer subtypes and patterns of metastasis on outcome. Breast Cancer Res Treat 150: 621-629, 2015.

25. Wu SY, Tan Y and Guan YS: Clinical features and prognosis of patients with first-episode liver metastasis of different molecular subtypes of breast cancer. Zhonghua Gan Zang Bing Za Zhi 24: 422-428, 2016 (In Chinese).

26. O'Conor CJ, Chen T, González I, Cao D and Peng Y: Cancer stem cells in triple-negative breast cancer: A potential target and prognostic marker. Biomark Med 12: 813-820, 2018.

27. Kaiser CG, Herold M, Krammer J, Baltzer P, Gajda M, Camara O, Schoenberg S, Kaiser WA and Dietzel M: Prognostic value of 'Prepectoral Edema' in MR-mammography. Anticancer Res 37: 1989-1995, 2017.

28. Baltzer PA, Dietzel M, Gajda, Camara O and Kaiser WA: A systematic comparison of two pulse sequences for edema assessment in MR-mammography. Eur J Radiol 81: 1500-1503, 2012.

29. Cheon H, Kim HJ, Lee SM, Cho SH, Shin KM, Kim GC, Park JY and Kim WH: Preoperative MRI features associated with lymphovascular invasion in node-negative invasive breast cancer: A propensity-matched analysis. J Magn Reson Imaging 46: 1037-1044, 2017.

30. Uematsu T, Kasami M and Watanabe J: Is evaluation of the presence of prepectoral edema on T2-weighted with fat-suppression $3 \mathrm{~T}$ breast MRI a simple and readily available noninvasive technique for estimation of prognosis in patients with breast cancer? Breast Cancer 21: 684-692, 2014.

This work is licensed under a Creative Commons Attribution-NonCommercial-NoDerivatives 4.0 International (CC BY-NC-ND 4.0) License. 